\title{
Antimicrobial activity of triple Antibiotic
}

\section{paste, Calcium hydroxide and Indextol against $E$. Faecalis - in vitro study}

\author{
Krasimir Hristov ${ }^{1}$, Rayna Gergova ${ }^{2}$, Adile Muhtarova ${ }^{2}$, Nataliya Gateva ${ }^{1}$
}

1. Department of Pediatric Dentistry, Faculty of Dental Medicine, Medical University- Sofia

2. Department of Microbiology, Faculty of Medicine, Medical University- Sofia

\section{Abstract}

Introduction: The aim of this study is to make an in vitro evaluation and comparison of the antimicrobial activity of the triple antibiotic paste, calcium hydroxide and indextol against E. faecalis. Materials and methods: Factory sealed packs of calcium hydroxide and indextol were used. The triple antibiotic paste in the desired concentration was prepared immediately before the experiment. Talcum powder mixed with saline was used as a control. A bacterial strain of $E$. faecalis was incubated in agar plates. Six wells were created in each plate - five peripheral and one central. The studied drugs were applied with sterile syringes in the peripheral wells, and in the central one - control with talcum powder in saline. One day after sample preparation, the inhibitory zone was measured from the edge of the well to the beginning of microbial growth. Results: The inhibitory zone is the largest in the samples treated with calcium hydroxide. In the other groups, the inhibition of microbial growth is significantly lower, as it decreases with the concentration of active ingredients in the triple antibiotic paste. The efficacy of Indextol against E. faecalis was comparable to that of TAP at a concentration of $100 \mu \mathrm{g} / \mathrm{mL}$. Conclusion: TAP at a concentration lower than $100 \mu \mathrm{g} / \mathrm{mL}$ is not effective in endodontic infections involving E. faecalis. With the exception of calcium hydroxide, none of the other intracanal dressings at the concentrations tested in the present study guaranteed good antimicrobial activity against $E$. faecalis.

Keywords: triple antibiotic paste, calcium hydroxide, antibacterial activity, E. faecalis 


\section{Introduction}

The main etiological cause of pulp necrosis and periapical lesions are the microorganisms and their byproducts [1]. There complete elimination is the most important factor for successful treatment of endodontic diseases [2]. Most of the microorganisms are removed by mechanical and chemical treatment of the root canals. Even with well-performed endodontic treatment, it has been shown that it is possible for microorganisms to persist in the dentinal tubules, the apical part of the root system, in anatomical features such as lateral canals, deltas and ramifications [2, 3].

Recent studies have focused on evaluating the efficacy of various root canals irrigants and dressings against Enterococcus faecalis, Staphylococcus aureus, and Candida albicans [4]. These microorganisms are one of the most resistant species inhabiting the oral cavity and they are considered to be the main cause of failure of root canal treatment [5]. In the case of positive cultures of infected root canals with chronic apical periodontitis, the most commonly isolated flora is polymicrobial with a predominance of E. faecalis [6]. E.faecalis can grow at $\mathrm{pH}=9.6$ and very low nutrient concentrations, tolerating $\mathrm{pH}$ values up to 11.9 . This microorganism has been shown to survive in the root canal and cause monoinfection or be part of a more complex canal microflora [6]. In addition, it has the ability to attach to the collagen of the dentinal tubules and thus remains in them [7]. This explains why its removal from the root canal with irrigation and antibacterial dressings is difficult.

$\mathrm{Ca}(\mathrm{OH}) 2$ has been used in the last 40 years in endodontics as an antimicrobial and antifungal agent due to its ability to dissolve necrotic tissues [8].

The main advantage of topically applied antibiotics compared to their use at the systemic level is that higher concentrations are achieved without side effects and reactions. However, antibiotic resistance is often demonstrated in bacteria isolated from root canals, with special attention being paid to Enteorococci, which show inherent or acquired through gene transfer resistance to antibiotics. The use of an interappointment dressings is thought to prevent the rapid growth of residual microorganisms in the root canal [9].

\section{Aim}

The aim of this study is to make an in vitro evaluation and comparison of the antimicrobial activity of the triple antibiotic paste, calcium hydroxide and Indextol against $E$. faecalis.

\section{Materials and methods}

Factory sealed packs of calcium hydroxide (Calcipast, Cerkamed, Stalowa Wola, Poland) and indextol (Indextol, Actavis Bulgaria, Sofia, Bulgaria) were used. The triple antibiotic paste (TAP) containing metronidazole, clindamycin and ciprofloxacin in a ratio of 1: 1: 1 was prepared immediately before the experiment. A paste at a concentration of $1000 \mu \mathrm{g} / \mathrm{mL}$ was used, from which samples with a concentration of $200,100,50$ and $25 \mu \mathrm{g} / \mathrm{mL}$ were prepared by serial dilution with polyethylene glycol. Talcum powder mixed with saline was used as a control.

A bacterial strain of $E$. faecalis was incubated in agar plates. Six wells were created in each plate with depth of $4 \mathrm{~mm}$ and a diameter of $6 \mathrm{~mm}$ - five peripheral and one central. The studied drugs were applied with sterile syringes in the peripheral wells, and in the central one - control with talcum powder in saline. One day after sample preparation, the inhibitory zone was measured from the edge of the well to the beginning of microbial growth. The experiment was repeated three times and the results were subjected to statistical analysis. 


\section{Results}

The sensitivity of the tested strain of E. faecalis to the indicated intracanal drugs in in vitro conditions was determined by agar well diffusion method (Figure 1).

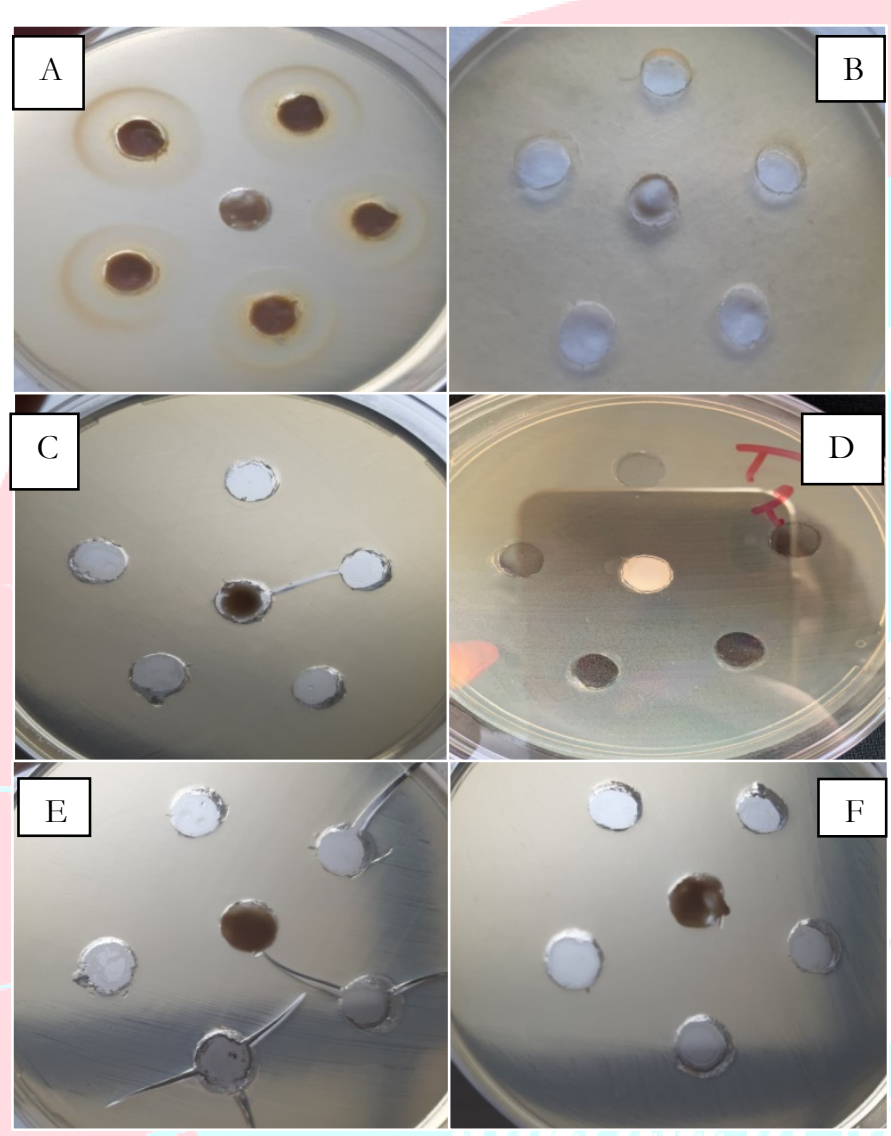

Figure 1. Agar petri dish with incubated $E$. faecalis and drugs placed in prepared wells.
A) Calcium hydroxide,

B) Indextol,

C) Triple antibiotic paste at concentration of $200 \mu \mathrm{g} / \mathrm{mL}$,

D) Triple antibiotic paste at a concentration of $100 \mu \mathrm{g} / \mathrm{mL}$,

E) Triple antibiotic paste at a concentration of $50 \mu \mathrm{g} / \mathrm{mL}$,

F) Triple antibiotic paste at a concentration of $25 \mu \mathrm{g} / \mathrm{mL}$, in the center - control with talcum powder.

Table 1 presents the results of the statistical analysis between the individual groups.

\begin{tabular}{|c|c|c|c|}
\hline & $\mathrm{M} \pm \mathrm{SD}$ & Min & Max \\
\hline Group $1\left(\mathrm{Ca}(\mathrm{OH})_{2}\right)$ & $11.167 \pm 0.699$ & 10.00 & 12.00 \\
\hline Group 2 (Indextol) & $1.3 \pm 0.535$ & 1.00 & 2.00 \\
\hline Group 3 (TAP $200 \mu \mathrm{g} / \mathrm{mL}$ ) & $1.867 \pm 0.776$ & 1.00 & 3.00 \\
\hline Group 4 (TAP $100 \mu \mathrm{g} / \mathrm{mL}$ ) & $1.167 \pm 0.379$ & 1.00 & 3.00 \\
\hline Group 5 (TAP $50 \mu \mathrm{g} / \mathrm{mL}$ ) & 0.00 & 0.00 & 0.00 \\
\hline Group $6($ TAP $25 \mu \mathrm{g} / \mathrm{mL})$ & 0.00 & 0.00 & 0.00 \\
\hline
\end{tabular}

Table 1. Results of the agar well diffusion test to determine the sensitivity of $E$. faecalis to the studied intracanal drugs.

The results show that the inhibitory zone is the largest in the samples treated with calcium hydroxide, the difference being statistically significant compared to the other study groups $(p<0.05)$. In the other groups, 
the inhibition of microbial growth is significantly lower, as it decreases with the concentration of active ingredients in the triple antibiotic paste. In the groups with the concentration of the triple antibiotic paste of 50 (Group 5) and 25 (Group 6) $\mu \mathrm{g} / \mathrm{mL}$ no inhibition of microbial growth was observed. The efficacy of Indextol (Group 2) against E. faecalis was comparable to that of TAP at a concentration of $100 \mu \mathrm{g} / \mathrm{mL}$ (Group 4) ( $p>0.05$ ). With the exception of calcium hydroxide, none of the other intracanal dressings at the concentrations tested in the present study guaranteed good antimicrobial activity against $E$. faecalis (Table 1).

\section{Discussion}

The success of endodontic therapy depends significantly on canal dressings and their antimicrobial properties [10]. They have an action that extends beyond the root canal lumen - in the dentinal tubules and the apical area [11].

The present study evaluates and compares in vitro the antimicrobial activity of triple antibiotic paste, calcium hydroxide and Indextol against E. faecalis.

E. faecalis was chosen as test microorganism in this study because it is easy to cultivate and has been used in other studies, which allows us to compare the results obtained $[12,13]$.

Calcium hydroxide is widely used in endodontics [14]. Its action is due to the high $\mathrm{pH}(11-12.5)$, as well as the ability of hydroxide anions to damage the cytoplasmic membrane, denature proteins, damage DNA and thus - kill bacterial cells [15].

It has low activity against E. faecalis, especially when this microorganism is included in the biofilm, as $\mathrm{E}$. faecalis is extremely resistant in alkaline environments $[1,14]$. The initial adhesion of microorganisms to the surface of the canal is the first step in biofilm formation and can lead to persistent or chronic infection [16]. The ability of E. faecalis to attach to collagen also improves at high $\mathrm{pH}$ values [17]. In this study, calcium hydroxide showed good antibacterial activity (Table 1), despite the reported in the literature lack of action against E. faecalis [18-20]. Probably the difference is due to the fact that in our study this microorganism was not included in the biofilm and it is not able to penetrate the dentinal tubules to protect it from the action of hydroxyl ions.

Combinations of antibiotics such as triple antibiotic paste (minocycline, ciprofloxacin and metronidazole) and double antibiotic paste (DAP) (ciprofloxacin and metronidazole) have been successfully used in cases with endodontic treatment failure associated with $\mathrm{E}$. faecalis [21, 22].

Taneja and Kumari have shown that TAP can be used as an alternative dressing in cases where calcium hydroxide fails to control the symptoms of persistent infection [23]. Minocycline acts against aerobic and anaerobic Gram-positive and Gram-negative bacteria, but it may cause tooth discoloration. TAP and DAP show similar efficacy against E. faecalis [24]. It has been found that the most appropriate concentration of TAP in terms of cytotoxicity and antibacterial activity for root canal disinfection in primary endodontic infection to be $39 \mu \mathrm{g} / \mathrm{mL}$ [25]. In the present study TAP with concentration of $50 \mu \mathrm{g} / \mathrm{mL}$ showed no antibacterial activity against $\mathrm{E}$. faecalis.

The third studied drug, indextol, belongs to the group of combined medications of corticosteroid and antibiotic, which are used for intracanal treatment due to their ability to relieve pain in acute and exacerbated apical periodontitis [26]. Application of this type of dressing for a period of 1 week does not effectively inhibit the growth of residual intracanal bacteria between visits. This is probably due to the fact that the two antibiotics in its composition act specifically against Gram-negative bacteria [15]. The present study showed that the efficacy of Indextol against E. faecalis was comparable to that of TAP at a concentration of $100 \mu \mathrm{g}$ / $\mathrm{mL}$ (Table 1, p>0.05). 


\section{Conclusion}

TAP at a concentration lower than $100 \mu \mathrm{g} / \mathrm{mL}$ is not effective in endodontic infections involving $\mathrm{E}$. faecalis. With the exception of calcium hydroxide, none of the other intracanal dressings at the concentrations tested in the present study guaranteed good antimicrobial activity against E. faecalis.

\section{References}

1. Stuart $\mathrm{CH}$, Schwartz $\mathrm{SA}$, Beeson TJ, et al. Enterococcus faecalis: its role in root canal treatment failure and current concepts in retreatment. J Endod 2006;32(2):93-98.

2. Singh G, Gupta I, Elshamy FMM, et al. In vitro comparison of antibacterial properties of bioceramicbased sealer, resin-based sealer and zinc oxide eugenol based sealer and two mineral trioxide aggregates. Eur J Dent 2016;10(3):366-369.

3. F JWKJT. In: I HKBLR, editor. Cohen's Pathways of the Pulp. 11. Missouri: Elsevier; 2016. p. 295-297. 4. Ruff ML, McClanahan SB, Babel BS. In vitro antifungal efficacy of four irrigants as a final rinse. J Endod 2006;32(4):331-333.

5. Park SY, Kang MK, Choi HW, et al. Comparative Analysis of Root Canal Filling Debris and Smear Layer Removal Efficacy Using Various Root Canal Activation Systems during Endodontic Retreatment. Medicina (Kaunas) 2020;56(11).

6. Barbosa-Ribeiro M, Arruda-Vasconcelos R, Louzada LM, et al. Microbiological analysis of endodontically treated teeth with apical periodontitis before and after endodontic retreatment. Clin Oral Investig 2021;25(4):2017-2027.

7. Dewi A, Upara C, Krongbaramee $\mathrm{T}$, et al. Optimal antimicrobial concentration of mixed antibiotic pastes in eliminating Enterococcus faecalis from root dentin. Aust Endod J 2021;47(2):273-280.

8. Blanscet ML, Tordik PA, Goodell GG. An agar diffusion comparison of the antimicrobial effect of calcium hydroxide at five different concentrations with three different vehicles. J Endod 2008;34(10):1246-1248.

9. Medina-Palacios SE, Vitales-Noyola M, López-González E, et al. Root canal microorganisms and their antibiotic susceptibility in patients with persistent endodontic infections, with and without clinical symptoms. Odontology 2021;109(3):596-604.

10. Sathorn $\mathrm{C}$, Parashos $\mathrm{P}$, Messer H. Australian endodontists' perceptions of single and multiple visit root canal treatment. Int Endod J 2009;42(9):811-818.

11. Lima SMF, de Pádua GM, Sousa M, et al. Antimicrobial peptide-based treatment for endodontic infections--biotechnological innovation in endodontics. Biotechnol Adv 2015;33(1):203-213.

12. Zancan RF, Calefi PHS, Borges MMB, et al. Antimicrobial activity of intracanal medications against both Enterococcus faecalis and Candida albicans biofilm. Microsc Res Tech 2019;82(5):494-500.

13. Morago A, Ruiz-Linares M, Ferrer-Luque CM, et al. Dentine tubule disinfection by different irrigation protocols. Microsc Res Tech 2019;82(5):558-563.

14. Zancan RF, Vivan RR, Milanda Lopes MR, et al. Antimicrobial Activity and Physicochemical Properties of Calcium Hydroxide Pastes Used as Intracanal Medication. J Endod 2016;42(12):1822-1828.

15. Tang G, Samaranayake LP, Yip HK. Molecular evaluation of residual endodontic microorganisms after instrumentation, irrigation and medication with either calcium hydroxide or Septomixine. Oral Dis 2004;10(6):389-397.

16. Baca $\mathrm{P}$, Junco P, Arias-Moliz MT, et al. Residual and antimicrobial activity of final irrigation protocols on Enterococcus faecalis biofilm in dentin. J Endod 2011;37(3):363-366.

17. Kayaoglu G, Erten $\mathrm{H}, \varnothing$ rstavik D. Growth at high pH increases Enterococcus faecalis adhesion to collagen. Int Endod J 2005;38(6):389-396. 
18. Delgado RJ, Gasparoto TH, Sipert CR, et al. Antimicrobial effects of calcium hydroxide and chlorhexidine on Enterococcus faecalis. J Endod 2010;36(8):1389-1393.

19. Mohammadi Z, Dummer PM. Properties and applications of calcium hydroxide in endodontics and dental traumatology. Int Endod J 2011;44(8):697-730.

20. Schäfer E, Bössmann K. Antimicrobial efficacy of chlorhexidine and two calcium hydroxide formulations against Enterococcus faecalis. J Endod 2005;31(1):53-56.

21. Ghabraei S, Bolhari B, Sabbagh MM, et al. Comparison of Antimicrobial Effects of Triple Antibiotic Paste and Calcium Hydroxide Mixed with 2\% Chlorhexidine as Intracanal Medicaments Against Enterococcus faecalis Biofilm. J Dent (Tehran) 2018;15(3):151-160.

22. Asnaashari M, Eghbal MJ, Sahba Yaghmayi A, et al. Comparison of Antibacterial Effects of Photodynamic Therapy, Modified Triple Antibiotic Paste and Calcium Hydroxide on Root Canals Infected With Enterococcus faecalis: An In Vitro Study. J Lasers Med Sci 2019;10(Suppl 1):S23-s29.

23. Taneja S, Kumari M. Use of triple antibiotic paste in the treatment of large periradicular lesions. J Investig Clin Dent 2012;3(1):72-76.

24. A AA, G HY, R LG. Inhibitory effect of gels loaded with a low concentration of antibiotics against biofilm formation by Enterococcus faecalis and Porphyromonas gingivalis. J Oral Sci 2015;57(3):213-218.

25. Namour M, Theys S. Pulp revascularization of immature permanent teeth: a review of the literature and a proposal of a new clinical protocol. ScientificWorldJournal 2014;2014:737503.

26. Bansal R, Jain A. Overview on the current antibiotic containing agents used in endodontics. N Am J Med Sci 2014;6(8):351-358.

\section{Corresponding author:}

Krasimir Hristov

Department of Pediatric Dentistry, Faculty of Dental Medicine,

Medical University- Sofia

1 Georgy Sofiyski Str,

Tel: +359883411743

e-mail: khristov87@gmail.com 